Meta

Journal des traducteurs

Translators' Journal

\title{
Traduire ce que les mots ne disent pas, mais ce qu'ils font
}

\section{Henri Meschonnic}

Volume 40, numéro 3, septembre 1995

La traduction, qu'est-ce à dire? Phénoménologies de la traduction

URI : https://id.erudit.org/iderudit/003640ar

DOI : https://doi.org/10.7202/003640ar

Aller au sommaire du numéro

Éditeur(s)

Les Presses de l'Université de Montréal

ISSN

0026-0452 (imprimé)

1492-1421 (numérique)

Découvrir la revue

Citer cet article

Meschonnic, H. (1995). Traduire ce que les mots ne disent pas, mais ce qu'ils font. Meta, 40(3), 514-517. https://doi.org/10.7202/003640ar

\section{Résumé de l'article}

Continuing his reflection and work on rhythm, the author shows how a poetics of translation can overcome the limits of a theory of sign based on the meaning/form dichotomy. As an alternative to this approach, which situates the poem and the translation in a logic of discontinuity, the author proposes that language and translation he reconsidered from the viewpoint of the continuity of discourse and the unity of rhythm. Consequently, translation consists in translating not what words say, hut what they do. As an example, the author applies this approach to the translation of the famous first two verses of Psalm 22. 


\title{
TRADUIRE CE QUE LES MOTS NE DISENT PAS, MAIS CE QU'ILS FONT
}

\author{
HENRI MESCHONNIC \\ Universilé de Paris VIIl. Vincennes. France
}

\begin{abstract}
Continuing his reflection and work on rhythm, the author show's how a poetics of translation can overcome the limits of a theory of sign hased on the meaninglform dichotomy. As an alternative to this approach, which situate's the poem and the transtation in a logic of discontinuity, the author proposes that language and translation be reconsidered from the viewpoint of the continuity of discourse and the unity of rhythm. Consequently, translation consists in translating not what words say. hut what they do. As an example, the author applies this approach to the translation of the famous first wo verses of Psalm 22.
\end{abstract}

Le problème théorique d'une poétique de la traduction, c'est-à-dire de la traduction des textes qui sont dits littéraires parce qu'ils sont une poétique en acte, c'est d'abord aujourd'hui de mettre fin au tourniquet dans lequel le pseudo-bon sens a généralement jusqu'ici enfermé la traduction des textes littéraires.

Ce tourniquet, c'est l'opposition des constituants du signel. Dans les limites du signe, où le signifié vaut pour la totalité du signe, et le signifiant est une forme escamotable-escamotée, maintenue comme un résidu, la traduction n'a le choix qu'entre traduire le sens, c'est ce qu'Eugene Nida a appelé l'équivalence dynamique, ou traduire, vouloir traduire, la forme. à la recherche de l'équivalence formelle. El dans les limites du signe, le bon sens même veut qu'on privilégie le sens, car il est bien vrai que tout le langage est pour le sens, pour le passage du sens, dans tous les sens.

Et la chose littéraire, bêtement, s'est laissée piéger, au point qu'on a cru de bonne foi que plus cette chose est littéraire, plus elle est consubstantielle à la forme dans le langage (comme si le sens, lui, était une substance - ce qui est étrange si, comme on peut le penser depuis Saussure, le langage tout entier est forme el non substance). Ainsi la poésie, couramment perçue comme un extrême de la chose littéraire, était naturellement vouée à être située dans le comble de la forme. À quoi se prêtaient les formes littéraires. Fixes ou non.

Mais si la pensée de ces choses pousse à penser ce qui n'est pas pensé dans les limites du signe, on peut concevoir, on est même obligé de concevoir ce que les concepts coutumiers enpêchent de penser. Par quoi la poétique devient un regard sur la théorie générale du langage. Le regard de l'oublié du signe.

D'où le signe apparaît avec ses limites. Alors que si on regarde le langage à partir du signe, il n'a pas de limites, il est tout le langage, sa nature même. Mais, vu du poème, le signe est le discontinu. Tout en lui et par lui est du discontinu - le discontinu entre langue et littérature, langue et culture : entre le langage et le corps. entre la voix et l'écrit. entre le mot et la chose, entre le son et le sens. D'un mot à l'autre comme d'une langue à l'autre. Et $\mathrm{j}$ 'en oublie. À partir de là. on tâche comme on peut, depuis longtemps, à rejoindre les deux bouts. On s'y essaie par l'expressivité, on court après la nature dans la déréliction du conventionnalisme, le poème est cette fameuse "hésitation prolongée entre le son et le sens".

Traduire s'est installé dans cette situation babélienne. On traduit du sens, des mots, des phrases, des langues. Comment faire autrement, comment concevoir qu'il en soit 
autrement ? Comment concevoir autre chose que l'antériorité du comprendre et de l'interpreter, par rapport au traduire ? Situation connue. Fin de l'histoire, tout est entendu.

Mais si l'intérêt de penser le langage continue, c'est bien qu'il y a de l'inconnu dans le langage. Et peut-être pas plus de progrès dans la pensée du langage qu'en art. Où la chose littéraire, et sa poussée au traduire, montreraient peut-être qu autant il y a des sciences du langage (en variables d'époque, d'ailleurs), il y aurait un art de penser le langage, que penser le langage, et penser le traduire, participeraient aussi d'un art. distinct de l'art du langage et de l'art de traduire.

Car ce qui est a penser est le continu dans le langage, et ce continu a besoin qu'on invente des concepts pour le penser. Pour comprendre, faire, et analyser le continu entre le corps et le langage, entre une langue et une littérature, entre une langue et une culture. En fait, il s'agit d'arriver à penser le discours avec des concepts du discours, pas les concepts de la langue.

C'est ici que le rythme, non plus dans son acception platonicienne, mais comme une organisation du mouvement de la parole dans l'écriture, comme système d'une subjectivation généralisée, ouvre une conceptualité qui n'est plus celle du signe. Et qui peut permettre de reconnaître ce qui a toujours existé, même si le signe ne permettait pas de le savoir. Dans cette écoute, il n'y a plus de double articulation du langage. - des phonèmes, des mots. Mais une sémantique sérielle, et ce qu'après Apollinaire, bien que dans un autre sens que lui, j'appelle des prosodies personnelles.

On n'a que trop confondu la littérature avec le récit. Il s'agit de reconnaître que ce qui définit spécifiquement chaque fois la chose littéraire, c'est le récitatif.

Et cette situation theorique nouvelle modifie le statut du traduire. Il ne s'agit plus de se précipiter vers l'équivalence formelle. Car l'équivalence formelle n'est qu'un effet de théorie du signe, et aussi liée à son opposé apparent, l'équivalence dynamique, que le son et le sens sont liés dans le signe.

C'est le système du discours qui est l'unité, pour la poétique. Toutes les autres unités sont d'un autre ordre, rhétoriques, ou culturelles. L'équivalence, la concordance ne sont plus lexicales, mais de l'ordre des paradigmes et des syntagmes d'une sémantique sérielle. Ainsi la poétique de la traduction n'est plus à situer dans un littéralisme, comme les gardiens du signe aiment tant à l'y reléguer ${ }^{2}$. Dans le mauvais rôle. Avec une bien mesquine idée de la poésie, aussi. Toute confondue avec le vers.

Un bonheur ne venant jamais seul, autant le traduire sort de l'opposition entre littéralisme et pragmatisme, en se situant dans le continu, autant il peut songer à renverser ce truisme inébranlable en apparence, selon lequel interpréter doit précéder traduire.

Car si on le regarde a partir du continu, on peut voir au travers le paralogisme qu il donnait pour une évidence, et qui, au contraire, apparaît maintenant comme l'une des conditions qui font les traductions mauvaises.

Car il ne s'agit pas, bien sûr, de laisser entendre cette absurdité qu'on pourrait traduire avant de comprendre. Mais de déceler que si on place la traduction comme terme d'une chaîne interprétative, on réalisera nécessairement une traduction qui ne fera plus ce que fait le texte qu'on traduit. Car le texte est porteur de la chaîne, et la traduction est seulement portée.

Le problème poétique est alors de faire que, tout en étant inévitablement au terme présent d'un savoir philologique et herméneutique, la poétique constitue la traduction plus que la philologie et l'herméneutique, pour que, poétique pour poétique, la traduction, tout simplement, texte pour texte, cohérence pour cohérence, systématicité pour systématicité, oralité pour oralité, soit à son tour non seulement portée mais porteuse.

C'est, empiriquement. ce qu'ont toujours fait les grandes traductions. celles qui durent. Elles ont toujours fait le contraire de ce qu'enseignent aujourd hui ceux qui 
enseignent qu'il faut effacer la distance linguistique, culturelle, historique: donner l'impression que le texte a été écrit dans la langue d'arrivée pour le ici-maintenant du lecteur ; effacer le traducteur qui devrait être transparent (et modeste). Sans voir que le cumul de tant de transparences a précisément pour résultat cette sorte particulière d'opacité qui fait que, prenant une de ces traductions entre les mains, on ne voit plus que ces effacements : une rhétorique au lieu d'une poétique, l'obscène de tout ce qui était caché qui se montre, et qui recouvre le texte, au lieu qu'on croyait le traduire. On a surtout traduit l'époque, ses clichés et ses goûts.

C'est donc seulement quand la poétique est une critique de la théorie générale du langage qu'on peut mieux voir que la traduction n'est pas seule. La croire autonome, c'est immédiatement la couper de la poétique restreinte des œuvres comme de la poétique générale du langage.

Aussitôt elle rejoint ce qu'elle $n$ 'a jamais réellement quitté, le signe et ses stratégies, particulièrement celles de certaines philosophies qui $n$ 'ont jamais su quoi faire d'un poème, sinon l'annexer, et noyer le traduire dans une généralisation du comprendre. L'essentialisation est alors un diffèrement indéfini de l'empirique. C'est-à-dire du continu.

Je ne prendrai ici qu'un exemple concret du continu dans le discours. D'où la traduction bifurque, loin de ce que disent les mots, selon leur sens, le sens du dictionnaire. vers ce qu'ils montrent mais ne disent pas, vers ce qu'ils font, qui est plus subtil que ce que la pragmatique contemporaine a cru mettre au jour.

II s'agit des deux premiers versets du psaume 22. Il y a bien eu assez de traductions, presque toutes semblables, pour qu'il vaille la peine d'une expérience qui nous donne à entendre ce qui résonne silencieusement dans ce texte. Le deuxième verset. fameux d'être cité par Jésus sur la croix dans Matthieu (XXVII, 46) : «Eli, Eli, lama sahakhthani ' c'est-à-dire Mon Dieu, mon Dieu, pourquoi m'as-tu abandonné ?"

Le premier verset traité comme une didascalie et une fantaisiste allusion à un air connu : «Pour le coryphée. Sur 'Biche de l'aurore' Psaume de David" (Dhorme): La Bible de Jérusalem: "Du maître de chant. Sur 'La Biche de l'aurore'. Psaume. De David"; la T.O.B. : "Du chef de chœur, sur 'Biche de l'aurore'. Psaume de David"; le Rabbinat de 1899: "Au chef des chantres. D'après l'Ayyelet Hachahar". Psaume de David": Chouraqui : "Au chorège. Sur: 'Biche de l'aube'. Chant. De David». On voit que les variantes $n$ 'y changent rien.

Quant au $2^{\mathrm{e}}$ verset, de la Septante à Jerôme (Deus Deus meus quare dereliquisti me), à la King James version (why hast thou forsaken me), à Luther (warumb hastu mich verlassen) en 1545, et à Buber et Rosenzweig, à Lemaistre de Sacy, au Rabbinat, à Segond, à la Bible de Jérusalem, à la T.O.B., à Chouraqui et dans les autres langues d'Europe où j'ai regardé, tous ont compris et traduit pourquoi m'as-tu abandonné.

Or le mot que tous traduisent, au premier verset, comme désignant le chef de chơur, lamenatséah, contient une allusion, entendue en hébreu, à netsah, la victoire, et une tradition ancienne de commentaire y interprétait une allusion à Dieu, qui la donne ; le mot qui désigne la biche, ayyélet (mais la «biche de l'aurore» est l'étoile du berger), a un écho au verset 20 du même psaume, eyalouti, "ma force" (puissance, vigueur).

J'ai donc traduit ${ }^{4}$, mais déjà saint Jérôme traduisait Victori (pro cervo matutino (anticum) :

De qui la victoire sur la force de l'aube un chant de David

Quant au $2^{\mathrm{c}}$ verset, Samson Raphael Hirsch's à ma connaissance, a été le seul à remarquer que le mot traduit par pourquoi, lama, a ce sens s'il est accentué sur la première syllabe, mais ici il est accentué sur la seconde, et n'a pas ce sens (il traduit "for what purposen) et je traduis: 
Mon Dieu mon Dieu a quoi m'as-tu abandonné

qui change le sens, anthropologiquement et théologiquement. Accessoirement, les deux Mon Dieu mon Dieu font un seul groupe, d'un seul souffle, sans ponctuation entre eux. comme font les autres, et l'impalpable du mode de signifier, affectivement, y modifie aussi non le sens mais la valeur.

Et c'est bien parce que traduire fait une poétique expérimentale - parce qu'il y a du retraduire - que la poétique de la traduction est nécessaire à la poétique des auvres autant que celle-ci lui est nécessaire. Le signe est vieux et sourd.

Et l'essentiel, avec le langage, est d'entendre, y compris tout ce que le sens, le règne du sens, nous empêche d'entendre.

Notes

1. Je developpe ce qui ici reste implicite dans Politique du rythme, politique du sujes. Verdier 1995, qui luimeme prend la suite de Crisique du rythme. Anshropologie historique du langage. Verdier. 1982.

2. Ce qui était justifié, quand l'unité était le mot (comme elle l'est dans le calque. de Chouraqui), non le rythme el la prosodie. C'est par un tel malentendu que Michel Ballard continue de voir dans ce que j"entends par la poetique de la traduction une défense du «litteralisme comme un absolu», dans De Cicéron a Benjamin (Presses Universitaires de Lille, 1992, p. 185), au point de prendre pour ma traduction (ibid.. p. 5 I) le mot à mot purement explicatif dont $j$ 'accompagne une transcription phonétique des cing premiers versets de la Genese, dans Pour la poérique II (Gallimard. 1973. p. 429), alors que j’en propose plus loin (p. 450) une traduction, qui n'a rien d'un mot à mot, après l'elude du texte hebreu et d'un centain nombre de traductions. Sur cette lecture hâtive, Michel Balland concluait à une apologie du litteralisme.

3. Mot à mot: Biche de l'Aurore.

4. Sur l'incitation de Claude Regy, dans le programme de l'Opera de Paris-Bastille. pour Jeanne d'Arc au bâcher, oratorio de Claudel et Honegger, novembre 1992. Les blancs sont ceux que j'ai employes dans mes traductions des Cinq Rouleaux (Gallimard, $3^{c}$ ed. 1995) et de Jona ef le signifiant errant (Gallimard, 1981 ).

5. The Psalms, Translation and Commentary by Rabbi Samson Raphael Hirsch. Feldheim Publishers, Jerusalem/New York, 1978 [1880]. 